
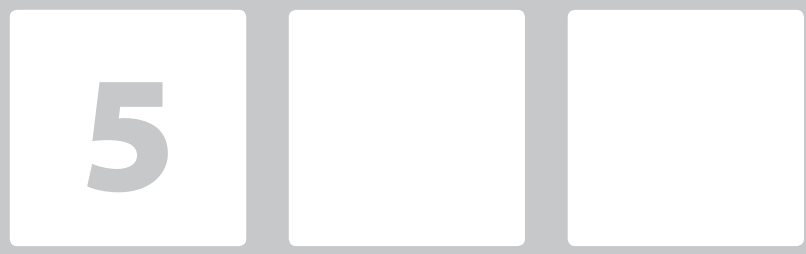

\title{
CARACTERIZAÇÃO DAS CAPACIDADES DE TECNOLOGIA DA INFORMAÇÃO: ESTUDO DE CASO EM UMA EM INSTITUIÇÃO DO ENSINO SUPERIOR
}

\section{Characterization of Information Technology Capabilities: Case Study in an Institution of Higher Education}

\author{
Josué Vitor de Medeiros Júnior \\ Doutor em Administração. Professor da Universidade \\ Federal do Rio Grande do Norte. Natal, RN. Brasil. \\ e-mail:josuevitor16@gmail.com
}

\section{Manoel Veras de Sousa Neto}

Doutor em Administração. Professor da Universidade

Federal do Rio Grande do Norte. Natal, RN. Brasil.

e-mail:manoel.veras@uol.com.br

\author{
Miguel Eduardo Moreno Anez \\ Pós-Doutorado em Administração. Professor da \\ Universidade Federal do Rio Grande do Norte. Natal, RN \\ e-mail:anez1957@yahoo.com.br
}

\section{RESUMO}

Este artigo busca identificar as capacidades de Tecnologia da Informação criadas pela Universidade Federal do Rio Grande do Norte (UFRN) durante a concepção, desenvolvimento, implantação e disseminação dos seus sistemas integrados de gestão entre os anos de 2004 e 2014. Tal sistema encontra-se em implantação em mais de 30 instituições do governo federal através de acordos de cooperação. Adotou-se como estratégia de pesquisa o estudo de caso e entrevistas semiestruturadas a partir de categorias de análise advindas da literatura. Enquanto resultado, foram identificadas as seguintes capacidades: capacidade técnica, habilidade organizacional em desenvolver aprimoramentos e correções nos sistemas; capacidade de relacionamento interno, habilidade organizacional em apoiar o uso efetivo dos sistemas por outras unidades organizacionais da instituição; e capacidade de cooperação externa, habilidade organizacional em transferir conhecimento para as instituições cooperadas. Espera-se que este trabalho contribua na compreensão do valor gerado pelas capacidades de Tl em contextos organizacionais diversos.

Palavras-chave: Capacidades Organizacionais; Capacidades de Tecnologia da Informação; Sistemas integrados de gestão.

\section{ABSTRACT}

This article aims to identify the IT capabilities created by the Federal University of Rio Grande do Norte (UFRN) during the design, development, implementation and dissemination of its integrated systems management between 2004 and 2014. This integrated system is currently being implemented in more than 30 federal government institutions through cooperation agreements. It was adopted as research strategy case study and semi-structured interviews from categories of analysis identified in the literature. As a result, the following relevant capabilities were identified: the technical capability, organizational ability to develop improvements and corrections in the systems; internal relationship capability, organizational ability to support the effective use of the systems by other organizational units; and external cooperation capability, organizational ability to transfer technological knowledge and business to the cooperative institutions. It is hoped that this research will contribute to the understanding of the value generated by IT capabilities in diverse organizational contexts.

Keywords: Organizational Capabilities; Information Technology Capabilities; Integrated Systems Management. 


\section{INTRODUÇÃO}

A Tecnologia da Informação (TI) é uma função cada vez mais indispensável para as organizações que buscam maior efetividade no ambiente em que atuam, e tem evoluído de uma orientação de suporte às atividades organizacionais para um papel mais estratégico e necessário à sobrevivência em longo prazo das organizações. Pesquisas na área de Sistemas de Informações (SI) buscam compreender como os recursos de Tecnologia da Informação, componentes tecnológicos usados para armazenar, processar e disseminar informações (NEVO; WADE, 2010) são relevantes para melhoria no desempenho operacional e financeiro das organizações (BHARADWAJ, 2000; WADE; HULLAND, 2004).

Em geral, tais recursos são vistos como commodities e disponíveis para aquisição no mercado, de maneira que não são considerados estratégicos para as organizações (MATA ET AL., 1995; CARR, 2003). Por outro lado, é cada vez mais disseminada a ideia de que são as capacidades de Tecnologia da Informação responsáveis mais diretamente por desempenhos superiores (LIANG ET AL., 2010). Do ponto de vista estratégico, organizações que constroem capacidades se valem desse importante mecanismo de criação de valor a partir de seus recursos para se adaptarem aos ambientes em que se encontram (TEECE ET AL., 1997; LEVINTHAL, 2000; MAKADOK, 2001).

Esta pesquisa trata especificamente das capacidades de Tecnologia da Informação, habilidades organizacionais compostas por rotinas que permitem à função de Tecnologia da Informação entregar serviços utilizando recursos complementares (BHARADWAJ, 2000; RAVICHANDRAN; LERTWONGSATIEN, 2005), tais como capacidade de relacionamento entre o setor de TI e demais unidades organizacionais; capacidade técnica e capacidade de infraestrutura (ROSS ET AL., 1996; BARNEY; CLARK, 2007; BHATT ET AL., 2010). Tais habilidades organizacionais têm um papel relevante enquanto responsáveis pela vantagem competitiva e desempenho superior das organizações, dado seu papel ativo em transformar os ativos tecnológicos essencialmente estáticos em elementos importantes para a formulação e implementação de estratégias.
Neste contexto, identificá-las permite direcionar melhorias e elaborar políticas de desenvolvimento destas habilidades organizacionais, através de iniciativas específicas (HARRISON ET AL., 2001; STIEGLITZ; HEINE, 2007; NEVO; WADE, 2010; NEVO; WADE, 2011), como reestruturação organizacional, treinamentos de pessoal e implementação de sistemas de apoio às atividades. Neste sentido, há uma lacuna na literatura quanto à identificação das capacidades de Tecnologia da Informação nas organizações, principalmente quando se trata de pesquisas realizadas na Administração Pública no Brasil.

Como em outros contextos organizacionais, a relevância da função de Tecnologia da Informação já é reconhecida nas instituições de ensino superior (SABHERWAL; KIRS, 1994). No entanto, há uma carência de estudos que tenham o foco em capacidades específicas de TI desenvolvidos nestas instituições, embora a literatura reconheça o papel de capacidades organizacionais gerais, seja, por exemplo, pela necessidade de adaptação ao ambiental externo (NAVARRO; GALLARDO, 2003), seja para identificar e avaliar recursos e capacidades destas instituições (PEREIRA; FORTE, 2004).

Isto dificulta a criação de visões de longo prazo das IES por não ter um alinhamento estratégico consistente que relacione as atividades diárias da função de Tecnologia da Informação com a estratégia organizacional, tendo em vista que a identificação das capacidades contribui no cumprimento deste papel (HELFAT, C. E.; WINTER, 2011).

Assim, este artigo se propõe e responder a seguinte questão: quais foram as capacidades de Tecnologia da Informação criadas na IES foco do caso e resultantes da trajetória de concepção, desenvolvimento, implantação e disseminação dos sistemas institucionais integrados de gestão desta organização?

Neste sentido, o presente artigo contribui para o avanço em estudos desta natureza ao possibilitar a construção efetiva destas capacidades em outras instituições de ensino superior, a partir do foco da gestão em políticas de desenvolvimento institucional e planejamentos de longo prazo, envolvendo mobilização e desenvolvimento de recursos (SIRMON; HITT; IRELAND, 2007).

Ademais, contribui ao adaptar critérios na identificação de características próprias das capa- 
cidades organizacionais, sejam estas dinâmicas ou operacionais, sem necessariamente enfatizar àquelas, amplamente exploradas na literatura, em detrimentos destas, relevantes para a execução das atividades diárias da organização (HELFAT; WINTER, 2011).

Além disto, trás o conceito para uma perspectiva mais próxima dos que se envolvem na execução da capacidade, facilitando a sua compreensão (SALVATO; RERUP, 2011), bem como propõe avanços nas definições das capacidades, ao contrapor as definições de capacidades de Tecnologia da Informação com as características do fenômeno a partir da realidade do caso pesquisado.

Os sistemas de informações foco deste trabalho foram inicialmente desenvolvidos a partir de 2004, em um contexto local, com o objetivo de automatizar e integrar atividades de natureza acadêmica, administrativa e posteriormente de recursos humanos, realizadas no âmbito da IES. Este objetivo foi atingido minimamente em 2008, de maneira que tais sistemas integrados passaram a dar apoio a todas as unidades organizacionais da instituição e vêm crescendo em escopo, atualizações e correções necessárias.

Em 2009, outras instituições de ensino superior bem como aquelas pertencentes à administração direta do Governo Federal tiveram interesse em implantar e utilizar tais sistemas, originando duas redes de cooperação: uma acadêmica (IFES) e outra da administração direta (CICLO), que têm crescido desde então. Atualmente são mais de 30 instituições parceiras em um modelo de cooperação caracterizado como de transferência tecnológica, de maneira que é responsabilidade da IES dar apoio às atualizações nos sistemas benéficas para as redes e treinamentos e esclarecimentos de dúvidas, enquanto que as instituições, ao terem acesso a este suporte e aos sistemas, têm a responsabilidade de implantá-los em seu âmbito interno, contando com equipe própria de Tecnologia da Informação ou contratando outros fornecedores para este fim.

\section{CAPACIDADES ORGANIZACIONAIS}

Historicamente, trabalhos seminais estabeleceram bases para definição de capacidades organizacionais ao darem ênfase em como um conjunto de recursos é utilizado nas organizações e não apenas pela mera posse deles (Andrews, 1971; Richardson, 1972; Penrose, 2006).

No âmbito da área de estratégia, a corrente de estudos denominada visão baseada em recursos trata as capacidades como um subconjunto ou tipo especial de recurso, embora reconheça que estas permitem a empresa aproveitar outros recursos que têm (Makadok, 2001; Barney; Clark, 2007). Assim, enquanto os recursos são ativos produtivos possuídos ou controlados pela organização, capacidades são compreendidas como habilidade organizacional em realizar uma atividade (Grant, 2010), tendo uma natureza intangível e sendo concretizadas como processos, rotinas, habilidades e conhecimento (Ulrich; Smallwood, 2004).

Nesse sentido, possuir uma capacidade implica dizer que a organização é capaz de mobilizar outros recursos físicos, humanos ou organizacionais, geralmente em combinação, através de processos organizacionais, provendo um significado de ação e atividade ao termo, com um sentido específico para organização: devem prover maior produtividade aos demais recursos, sendo assim vistas de maneira abstrata como bens intermediários (Amit; Schoemaker, 1993).

Dessa maneira, capacidade não existe em si mesma, caso não hajam estoques de recursos que justifiquem ações sobre estes. São, portanto, limitadas pelo conjunto de recursos e outras capacidades que a organização possui, implicando em um sentido de interdependência e necessidade de coerência na gestão das diversas capacidades em uma organização (Leinwand; Mainardi, 2010). Sob este enfoque, capacidades são bastantes específicas para a organização ao qual pertencem, e assim de difícil transferência e imitação, importante atributo para considerá-la como um fator estratégico.

Uma questão levantada diz respeito à maneira com que o conceito é tratado na literatura, muitas vezes em uma conotação abstrata e longe da realida- 
de dos indivíduos que realizam atividades diárias na organização (Salvato; Rerup, 2011). Ao lidar com esta dificuldade, alguns autores como Dosi et al. (2000), Eisenhardt e Martin (2000) definiram o termo como o know-how que permite às organizações realizar atividades como criação de produtos, fornecimento de serviços, desenvolvimento de novos produtos e serviços, aquisições e fusões com outras organizações.

Assim, o desempenho destas atividades sinaliza a existência de uma capacidade, tornando-a concretizada no dia-dia das organizações, conforme atestado por Pregelj (2013). Além disso, dão a ela um propósito organizacional claro e busca por um resultado intencional e específico (Amit; Schoemaker, 1993). Em linhas gerais, capacidades têm como propósito principal a criação, sustentação ou melhoria de atividades existentes (Pregelj, 2013).

De modo geral, capacidade organizacional pode ser identificada como uma habilidade da organização, e não limitada a indivíduos, com tarefas coordenadas e formada por rotinas organizacionais, apresentando desempenho em suas atividades como minimamente satisfatório e confiável e que tenham um resultado claro e específico. Cada um destes pressupostos conceituais será apresentado a seguir.

Afirmar que uma organização tem uma capacidade implica dizer que possui a habilidade em realizar uma atividade de maneira confiável e minimamente satisfatória (Helfat; Winter, 2011). Esta confiabilidade se reflete no padrão do desempenho demonstrado pela realização da capacidade, em contraste com uma atividade ad hoc realizada de maneira não-rotineira (Winter, 2003).

Também significa que o resultado da atividade é reconhecido como tal e funciona como intencionado, embora possa existir bastante possibilidade de melhoria neste desempenho funcional (Helfat; Peteraf, 2003). Assim, um conjunto de atividades só pode ser considerado como resultante de uma capacidade quando de reconhecido 'sucesso' em seu desempenho e resultados em diversas situações, de maneira que a organização possa reproduzi-la (Schreyögg; Kliesch-Eberl, 2007).

Este importante conceito pode ainda ser definido como habilidade de uma organização em realizar um conjunto de tarefas coordenadas, usando recursos organizacionais, com o propósito de alcançar um resultado final particular (Helfat; Peteraf, 2003). Exige, portanto, não apenas a execução de tarefas ou atividades, mas sua efetiva coordenação.

Quando se analisa o aspecto empírico das capacidades, pode-se reconhecer a existência destas nas organizações a partir do comportamento repetido e padronizado que a caracteriza. Neste sentido, manter uma capacidade implica no exercício constante de suas atividades (rotineiras) por parte dos membros de uma organização. Este aspecto faz com que muitos autores considerem rotinas como blocos de construção das capacidades, vislumbrando-as como formadas por rotinas componentes de mais baixo nível (COLLIS, 1994; DOSI ET AL., 2000; WINTER, 2000; HELFAT; PETERAF, 2003). Neste sentido, rotinas representam a concretização das capacidades.

Capacidades são vistas então como habilidades organizacionais para realizar e sustentar um conjunto de rotinas ou uma macro rotina. Esta relação entre os dois conceitos foi inicialmente apresentada em Nelson e Winter (1982), no contexto da economia evolucionária, cuja definição de rotina é central no modelo proposto por estes autores: padrões de comportamentos organizacionais regulares e previsíveis.

Mais recentemente, rotinas são conceituadas como padrões repetidos e reconhecidos de ações interdependentes realizadas por múltiplos atores (Feldman; Pentland, 2003). A repetição das rotinas permite que se visualize a performance destas através de suas múltiplas instâncias.

Tendo em vista a hierarquia de capacidades de maior ou menor nível de abstração em relação às atividades mais concretas realizadas na organização, é possível classificá-la de acordo com seus objetivos ao agir sobre outros recursos e capacidades.

Uma capacidade é dita operacional, de zero ordem ou ordinária, quando se relaciona diretamente aos produtos e serviços oferecidos por uma organização, bem como às suas receitas e despesas, impactando diretamente no desempenho organizacional. Permite à organização sobreviver, através de suas operações básicas, de maneira contínua, usando aproximadamente as mesmas técnicas na mesma escala para dar suporte a produtos e serviços existentes para a mesma população de clientes (Helfat; Winter, 2011). 
Por outro lado, capacidades dinâmicas ou de segunda ordem são aquelas que possibilitam mudanças nas capacidades ordinárias, agindo sobre elas (Zollo; Winter, 2002; Winter, 2003). São ainda definidas como a habilidade da organização em integrar, construir e reconfigurar competências internas e externas para lidar com ambientes de rápida mudança (Teece et al., 1997). Possibilitam assim, que organizações intencionalmente criem, estendam ou modifiquem sua base de recursos (Helfat et al., 2009).

As organizações devem manter um equilíbrio quanto ao desenvolvimento e manutenção destes dois tipos de capacidades, do contrário, decisões podem levar à erosão das capacidades (RAHMANDAD; REPENNING, 2016). Enquanto faz-se necessário manter um desempenho superior baseado nas atividades que caracterizam as operações diárias, como produção, distribuição, vendas, mecanismos de precificação e comercialização, as organizações precisam também sistematicamente adaptar suas entidades de mais baixo nível, como outras capacidades e rotinas operacionais, ao ambiente dinâmico, através de atividades de pesquisa e desenvolvimento, fusões e aquisições, por exemplo.

\section{CAPACIDADES DE TECNOLOGIA DA INFORMAÇÃO}

As capacidades de Tecnologia da Informação têm sido reconhecidas na literatura como fator mais apropriado de contribuição dos recursos de Sistemas de Informação no desempenho organizacional e vantagem competitiva (LIANG ET AL., 2010). Estas podem ser definidas como as habilidades e práticas que se complementam, realizadas pela organização para mobilizar e desenvolver recursos baseados na Tecnologia da Informação em combinação com outros recursos e capacidades (BHARADWAJ, 2000; ARAL; WEILL, 2007).

Esta definição explicita a necessidade das organizações em realizar atividades equilibradas na gestão de seus recursos (ou ativos) de Tecnologia da Informação: é na maneira que utilizam estes recursos a partir de combinações, alocações e integrações, que pode haver um impacto na efetividade organizacional (MAKADOK, 2001; SIRMON ET AL., 2007).
Em uma perspectiva mais relacionada à função da Tecnologia da Informação, capacidades são desenvolvidas ao longo do tempo através do desenvolvimento, avaliações e refinamentos de rotinas que possibilitam a esta área fornecer serviços baseados na Tecnologia da Informação para a organização (RAVICHANDRAN; LERTWONGSATIEN, 2005). Isto demonstra a importância no refinamento de rotinas organizacionais relacionadas às atividades realizadas pelo setor de Tecnologia da Informação, resultando em maior desempenho destas. Ao detalhar tais atividades, Ross et al. (1996) define capacidade de Tecnologia da Informação como a habilidade organizacional em identificar sistemas que atendam às necessidades organizacionais, desenvolvê-los de maneira efetiva em custos e oferecer suporte em longo prazo para eles.

Assim, pode-se considerar que este conceito envolve complexos conjuntos de recursos relacionados a Tecnologia da Informação, habilidades e conhecimentos, exercitados através de processos organizacionais que possibilitem à organização coordenar atividades e fazer uso dos ativos de Tecnologia da Informação para prover os resultados desejados (STOEL; MUHANNA, 2009).

Em geral, capacidades de Tecnologia da Informação podem ser abordadas sob uma perspectiva interna ou externa (HULLAND ET AL., 2007; STOEL; MUHANNA, 2009). Capacidades focadas externamente, e geralmente caracterizadas como dinâmicas, são habilidades e conhecimentos que apoiam a organização na identificação de mudanças ambientais e adaptações em tempo a estas alterações, bem como antecipação de tendências. Podem ser exemplificadas neste contexto a capacidade de relacionamento externo, em responder ao ambiente, de planejamento e mudança, e de pesquisa e desenvolvimento.

A capacidade de relacionamento externo é a habilidade organizacional em gerenciar relações entre a função de Tecnologia da Informação e atores externos à organização, como fornecedores, clientes ou parceiros (WADE; HULLAND, 2004). No caso dos fornecedores, o relacionamento deve favorecer a seleção e contratação efetiva destes, além de assegurar a manutenção de um padrão de desempenho e de comunicação para resolução de conflitos e problemas (FEENY; WILLCOCKS, 1998). Quanto 
aos clientes, são construídas a partir de interações repetidas com estes que se tornam efetivas em custo ao longo do tempo. Isto ocorre pelo aprendizado da função de Tecnologia da Informação sobre aspectos como infraestrutura e incorporação do sistema de informações ao ambiente organizacional do cliente (ETHIRAJ ET AL., 2005).

A capacidade em responder ao ambiente envolve a captura sistemática de informações de fontes externas bem como a disseminação desta inteligência dentro da organização, possibilitando que esta responda rapidamente ao aprendizado gerado (WADE; HULLAND, 2004). Neste contexto, as organizações podem desenvolver mecanismos de coordenação de atividades para entender, colaborar e responder às necessidades de seus clientes e a partir das informações advindas do relacionamento com eles fornecer respostas apropriadas às suas necessidades (RAPP ET AL., 2010).

A capacidade de planejamento e mudança em Tecnologia da Informação se relaciona a habilidade em antecipar crescimento e mudanças futuras das necessidades organizacionais, através dos ativos de Tecnologia da Informação, e selecionar plataformas de hardware, software, banco de dados e redes que possam acomodar estas mudanças. Inclui ainda a efetiva gestão destas mudanças e crescimento da tecnologia resultante (BHARADWAJ, 2000; WADE; HULLAND, 2004). Esta é uma importante capacidade, já que possibilita a identificação das prioridades organizacionais e a garantia de que os objetivos e iniciativas da função de Tecnologia da Informação estejam alinhados a estas prioridades, possibilitando, por exemplo, a identificação e construção de aplicações de TI estratégicas (RAVICHANDRAN; LERTWONGSATIEN, 2005).

Essencialmente voltada para o futuro, a capacidade de pesquisa e desenvolvimento em Tecnologia da Informação refere-se a habilidade organizacional em realizar experimentações com novas tecnologias, procurando adequá-las à realidade organizacional e mensurando possíveis benefícios, além de manter um nível de alerta para tendências e tecnologias emergentes que permita à organização rapidamente ter vantagem caso adote estes avanços (WADE; HULLAND, 2004). Possibilita que tecnologias não se tornem obsoletas e comprometam o crescimento organizacional, bem como flexibilidade às escolhas estratégicas futuras da organização.

Capacidades direcionadas para o ambiente interno ajudam a organização a prover produtos e serviços confiáveis e minimizar custos desnecessários, refletindo as operações internas da organização. São exemplos destas a capacidade de relacionamento interno, técnica, de operações efetivas de TI e de infraestrutura flexível.

A capacidade de relacionamento interno refere-se à habilidade de promover um relacionamento duradouro entre a função de Tecnologia da Informação da organização e os usuários da tecnologia, comumente gestores das demais unidades organizacionais (BHARADWAJ ET AL., 1999). Baseia-se no estabelecimento de um diálogo contínuo entre a função de TI e a comunidade de usuários, implicando na construção de confiança e compartilhamento de riscos (BHATT; GROVER, 2005), esforços para desenvolver a compreensão do potencial da TI pelos usuários, além de possibilitar que usuários e especialistas em TI trabalhem juntos, garantindo a satisfação e sentimento de propriedade por parte daqueles. Resulta em confiança mútua, harmonia de propósitos e comunicação facilitada entre estes dois atores (FEENY; WILLCOCKS, 1998).

A capacidade técnica em TI refere-se ao know-how necessário para projetar e desenvolver sistemas de informações efetivos usando a tecnologia disponível, bem como usar, aplicar e gerenciar este conhecimento para produzir bens e serviços (PICCOLI; IVES, 2005; BARNEY; CLARK, 2007; HULLAND ET AL., 2007). Envolve o conhecimento sobre linguagens de programação e de banco de dados, ambientes de desenvolvimento, padrões arquiteturais, protocolos de comunicação e sistemas operacionais.

Tipicamente, como as habilidades técnicas são explicitamente codificadas e devido a mobilidade dos profissionais de Tecnologia da Informação entre organizações, esta capacidade não é considerada como responsável pela vantagem competitiva nas organizações, já que não obedece ao pressuposto de heterogeneidade de recursos (MATA ET AL., 1995; BARNEY; CLARK, 2007). Por outro lado, algumas habilidades são difíceis de serem imitadas, como o domínio e adoção dos ativos de conhecimento em nível corporativo, necessários para codificação das 
regras de negócios de uma organização e habilidades de integração tecnológica, específicas da realidade organizacional (WADE; HULLAND, 2004).

Diante da penetração atual da Tecnologia da Informação nos processos e estrutura organizacional, falhas na disponibilidade e desempenho dos sistemas podem resultar em grandes perdas financeiras. Assim, para as organizações, a própria efetividade do negócio depende das operações confiáveis e eficientes realizadas pelos seus ativos de Tecnologia da Informação. Nesta visão, a capacidade de operações de TI efetivas se relaciona com a habilidade de fornecer operações de TI efetivas em custos e eficientes, de maneira contínua, evitando altos custos excedentes e persistentes, downtimes desnecessários e outras falhas nos sistemas que possam impactar em sua disponibilidade (ROSS ET AL., 1996; WADE; HULLAND, 2004).
Por fim, a capacidade de infraestrutura de TI flexível se refere ao nível de compartilhamento e reutilização dos recursos componentes desta infraestrutura, possibilitando que sejam adotadas para diversos fins. Organizações que possuem esta capacidade adaptam sua infraestrutura de Tecnologia da Informação a mudanças nos requisitos de negócios através da escalabilidade ou alcance desta estrutura e modularidade ou extensão com o qual os dados são disponibilizados (KEEN, 1993; DUNCAN, 1995; FINK; NEUMANN, 2009), permitindo entregar soluções técnicas de maneira rápida e efetiva (BYRD; TURNER, 2001). Além disso, esta flexibilidade permite que novas tecnologias sejam integradas a estrutura já existente (RAVICHANDRAN; LERTWONGSATIEN, 2005).

O quadro 1 a seguir apresenta os diferentes tipos de capacidades bem como as referências adotadas para explicar cada uma.

Quadro 1 Capacidades de Tecnologia da Informação relevantes identificadas na literatura.

\begin{tabular}{|c|c|c|}
\hline Capacidade De Ti & Definição & Referências \\
\hline $\begin{array}{l}\text { Capacidade de } \\
\text { Relacionamento Externo }\end{array}$ & $\begin{array}{l}\text { Habilidade organizacional em gerenciar relações entre a função de } \\
\text { Tl e clientes, parceiros ou fornecedores, externos à organização. }\end{array}$ & $\begin{array}{l}\text { (DAY, 1994; ETHIRAJ et al., 2005; FEENY; } \\
\text { WILLCOCKS, 1998; WADE; HULLAND, } \\
\text { 2004) }\end{array}$ \\
\hline $\begin{array}{l}\text { Capacidade de } \\
\text { Relacionamento Interno }\end{array}$ & $\begin{array}{l}\text { Refere-se a capacidade em promover um relacionamento } \\
\text { duradouro entre os especialistas na função de TI da organização } \\
\text { e os usuários da tecnologia, comumente gestores de unidades } \\
\text { organizacionais. }\end{array}$ & $\begin{array}{l}\text { (BHARADWAJ, A. et al., 1999; BHATT, G. } \\
\text { D.; GROVER, 2005; FEENY; WILLCOCKS, } \\
\text { 1998) }\end{array}$ \\
\hline $\begin{array}{l}\text { Capacidade em } \\
\text { responder ao ambiente }\end{array}$ & $\begin{array}{l}\text { Captura sistemática de informações de fontes externas bem como } \\
\text { a disseminação desta inteligência dentro da organização, possibili- } \\
\text { tando que esta responda rapidamente a este aprendizado }\end{array}$ & $\begin{array}{l}\text { (BHATT, G., 2000; COHEN; LEVINTHAL, } \\
\text { 1990; RAPP et al., 2010; WADE; HULLAND, } \\
\text { 2004) }\end{array}$ \\
\hline $\begin{array}{l}\text { Capacidade de } \\
\text { Planejamento e } \\
\text { Mudança em Tl }\end{array}$ & $\begin{array}{l}\text { Habilidade em antecipar mudanças futuras das necessidades orga- } \\
\text { nizacionais e selecionar plataformas de TI que possam acomodar } \\
\text { estas mudanças, além de efetivamente gerenciar estas mudanças e } \\
\text { crescimento da tecnologia resultante }\end{array}$ & $\begin{array}{l}\text { (BHARADWAJ, A., 2000; RAVICHAN- } \\
\text { DRAN; LERTWONGSATIEN, 2005; WADE; } \\
\text { HULLAND, 2004) }\end{array}$ \\
\hline Capacidade Técnica em TI & $\begin{array}{l}\text { Know-how necessário em projetar e desenvolver sistemas de infor- } \\
\text { mações efetivos usando a tecnologia disponível, bem como usar, } \\
\text { aplicar e gerenciar este conhecimento para produzir bens e serviços }\end{array}$ & $\begin{array}{l}\text { (BARNEY; CLARK, 2007; HULLAND et al., } \\
\text { 2007; MATA et al., 1995; PICCOLI; IVES, } \\
\text { 2005) }\end{array}$ \\
\hline $\begin{array}{l}\text { Capacidade de Pesquisa e } \\
\text { Desenvolvimento em } \mathrm{Tl}\end{array}$ & $\begin{array}{l}\text { Habilidade organizacional em realizar experimentações com novas } \\
\text { tecnologias e manter um nível de alerta para tendências e tecnolo- } \\
\text { gias emergentes }\end{array}$ & (WADE; HULLAND, 2004) \\
\hline $\begin{array}{l}\text { Capacidade de } \\
\text { Operações de TI Efetivas }\end{array}$ & $\begin{array}{l}\text { Habilidade de fornecer operações de Tl efetivas em custos e } \\
\text { eficientes, de maneira contínua, evitando altos custos excedentes e } \\
\text { persistentes, downtimes desnecessários e outras falhas nos sistemas }\end{array}$ & $\begin{array}{l}\text { (ROSS et al., 1996; WADE; HULLAND, } \\
\text { 2004) }\end{array}$ \\
\hline $\begin{array}{l}\text { Capacidade de } \\
\text { Infraestrutura de TI Flexível }\end{array}$ & $\begin{array}{l}\text { Refere-se à habilidade organizacional da infra de TI adaptar-se a } \\
\text { requisitos de negócios em mudança através da escalabilidade (ex- } \\
\text { pansão de capacidades) ou modularidade (incorporação/exclusão } \\
\text { de elementos da infra) }\end{array}$ & $\begin{array}{l}\text { (BHARADWAJ, A., 2000; BYRD; TURNER, } \\
\text { 2000; FINK; NEUMANN, 2009) }\end{array}$ \\
\hline
\end{tabular}

Fonte: Elaborado pelo autor (2014). 


\section{METODOLOGIA}

Esta pesquisa caracteriza-se como de natureza qualitativa e se classifica principalmente como descritiva (Gil, 2002), ao focar na descrição das características do fenômeno estudado, tornando-o mais explícito. Adotou a estratégia de pesquisa de estudo de caso, dada a necessidade em se detalhar e compreender um fenômeno específico, em um contexto particular, focada numa situação em profundidade: o contexto da trajetória de concepção, implantação e disseminação dos sistemas integrados de gestão de uma IES (GODOY, 2006).

Justifica-se ainda em tal estratégia por buscar responder questões do tipo "como" e "por quê", por não exigir controles de eventos comportamentais, mas estar focado em eventos contemporâneos sem preocupar-se em separar o fenômeno de seu contexto (YIN, 2003), características que se adequam à compreensão de capacidades de Tecnologia da Informação I, só possível de ser compreendida quando considerada em um contexto organizacional específico (Montealegre, 2002).

O estudo de caso único relacionado à trajetória dos sistemas integrados foi selecionado pela relevância para o contexto regional em que foi concebido, implantado e disseminado, já que se trata de um caso bem-sucedido de um sistema de informação, tendo sua tecnologia e conhecimento transferidos para outras cidades e regiões do país, a partir de um estado nordestino do Brasil.

A trajetória de disseminação destes sistemas também demonstra a sua raridade, resultante de um cenário favorável tanto internamente na instituição, que incentivou e investiu nos sistemas integrados, quanto fora dela, em virtude de iniciativas fracassadas de outras organizações em desenvolver sistemas similares.

Por fim, vale ressaltar as características do caso que o credenciam como relevante, já que através dele foi possível identificar aspectos possíveis de serem conhecidos a respeito de capacidades de Tecnologia da Informação relevantes.

A pesquisa foi realizada em duas etapas distintas. $\mathrm{Na}$ primeira, buscou-se identificar uma cronologia a respeito dos principais eventos relacionados a concepção, implantação e disseminação dos sistemas integrados, desde 1999 até o ano de 2013, através de entrevistas com participantes diretos deste processo. O objetivo desta fase foi compreender eventos relevantes que pudessem servir como âncoras e oferecer suporte aos questionamentos da fase subsequente.

$\mathrm{Na}$ segunda etapa, foram apresentadas as descrições conceituais de oito capacidades de TI, com base na literatura, para que os entrevistados pudessem reconhecer quais poderiam caracterizar-se como capacidades organizacionais e relevantes na trajetória dos sistemas integrados, tendo em vista os cinco questionamentos a seguir baseados em principalmente em (NELSON; WINTER, 1982; DOSI ET AL., 2000; WINTER, 2000; HELFAT; PETERAF, 2003): "é uma habilidade organizacional?", "é formada por rotinas?", "possui tarefas coordenadas?", "possui desempenho confiável e minimamente satisfatório?" e "tem um resultado específico e reconhecido?".

Ao serem identificadas as capacidades, mediante a resposta afirmativa a todas estas questões por todos os entrevistados, buscou-se compreender a definição que tinham sobre cada uma, convertendo a definição formalmente da literatura em uma definição contextualizada. Isso possibilitou sugerir avanços na definição do fenômeno na literatura ao contrapor com os dados capturados no caso.

Ademais, foi construído um gráfico de um importante indicador de desempenho da atividade relacionada à capacidade (prazo de atendimento) em relação ao tempo, a partir de dados secundários. Este indicador foi selecionado mediante reconhecimento dos entrevistados e acessibilidade do pesquisador aos dados quantitativos longitudinais sobre sua ocorrência. O objetivo em se desenhar gráficos para cada capacidade, reconhecida como relevante na trajetória dos sistemas integrados, foi atestar que elas evoluíram ao longo do tempo.

Os dados da pesquisa foram capturados através de entrevistas semiestruturadas, com duração entre cinquenta minutos e uma hora e quarenta minutos cada uma. Os dados secundários, usados principalmente para confirmação de datas e conteúdo, foram capturados a partir de documentos como apresentações, projetos, documentos normativos, vídeos e dados capturados dos sistemas de informações, principalmente o repositório de documentação e 
o sistema de controle do setor de Tecnologia da Informação.

As entrevistas ocorreram entre os meses de outubro e dezembro de 2013 , tendo sido realizadas 08 entrevistas com membros do setor de Tecnologia da Informação, pró-reitores e responsáveis pelos projetos de cooperações nas instituições de ensino da rede IFES para levantamento histórico e 10 entrevistas com membros do setor de Tecnologia da Informação para identificação das capacidades de Tecnologia da Informação.

Para análise e organização dos dados coletados, foi utilizado o software de análise de dados qualitativos NVivo versão 10, no intuito de apoiar a realização de transcrições e codificação dos dados. Esta técnica central adotada durante a análise de dados diz respeito a indexação ou categorização de textos visando estabelecer uma estrutura de ideias temáticas sobre ele (GIBBS, 2009).

As categorias criadas foram baseadas na literatura, e portanto, pré-definidas, e relacionadas a duas dimensões: tipos de capacidades de Tecnologia da Informação e características das capacidades. O texto relacionado a cada categoria foi identificado a partir de uma análise linha a linha dos dados transcritos a partir das entrevistas, principalmente.

\section{CAPACIDADES DE TECNOLOGIA DA INFORMAÇÃO IDENTIFICADAS}

Além dos marcos históricos importantes na trajetória dos sistemas integrados, foram identificadas as capacidades reconhecidas como relevantes neste processo. A partir das oito capacidades de Tecnologia da Informação da literatura, foram identificadas três capacidades identificadas como uma habilidade disseminada organizacionalmente (e não limitada a indivíduos), formada por rotinas organizacionais (através de padrões na sequência de ações), com tarefas coordenadas (e não apenas executadas), apresentando desempenho em suas atividades como minimamente satisfatório e confiável e que tenham um resultado claro e específico, pressupostos conceituais adotados para se caracterizar uma capacidade organizacional. A tabela abaixo representa o nível de reconhecimento de cada uma das capacidades pelos entrevistados.

Quadro 2 Identificação das capacidades de Tecnologia da Informação pelos entrevistados

\begin{tabular}{|c|c|c|c|c|c|c|c|c|c|c|}
\hline Capacidades & E1 & E2 & E3 & E4 & E5 & E6 & E7 & E8 & E9 & E10 \\
\hline Relacionamento Externo & $\boldsymbol{*}$ & $*$ & $\boldsymbol{*}$ & $\boldsymbol{x}$ & $\boldsymbol{*}$ & $\boldsymbol{*}$ & $\boldsymbol{*}$ & $\boldsymbol{x}$ & $\boldsymbol{*}$ & $\boldsymbol{*}$ \\
\hline Responder ao Ambiente & $\boldsymbol{*}$ & & & & & & & $\boldsymbol{*}$ & & \\
\hline Relacionamento Interno & $\boldsymbol{*}$ & $\boldsymbol{*}$ & $\boldsymbol{*}$ & $\boldsymbol{*}$ & $\boldsymbol{x}$ & $*$ & $\boldsymbol{x}$ & $\boldsymbol{*}$ & $\boldsymbol{x}$ & $*$ \\
\hline Planejamento e Mudança & & & & & & & $\boldsymbol{*}$ & & & $\boldsymbol{*}$ \\
\hline Técnica & $\boldsymbol{*}$ & $*$ & $\boldsymbol{x}$ & $\boldsymbol{*}$ & $\boldsymbol{*}$ & $\boldsymbol{*}$ & $\boldsymbol{*}$ & $\boldsymbol{*}$ & $\boldsymbol{*}$ & $\boldsymbol{*}$ \\
\hline Pesquisa e Desenvolvimento & & $*$ & & & & & $\boldsymbol{*}$ & $\boldsymbol{*}$ & & $\boldsymbol{*}$ \\
\hline Operações de TI Efetivas & & $*$ & $\boldsymbol{x}$ & $\boldsymbol{*}$ & $\boldsymbol{x}$ & $\boldsymbol{x}$ & & & $\boldsymbol{*}$ & \\
\hline Infraestrutura de TI Flexível & $\boldsymbol{*}$ & $\boldsymbol{*}$ & & & $\boldsymbol{x}$ & * & $\boldsymbol{*}$ & $\boldsymbol{*}$ & * & \\
\hline
\end{tabular}

Fonte: Elaborado pelo autor (2014).

\subsection{Capacidade Técnica}

Segundo os entrevistados, esta é a principal capacidade responsável pela trajetória bem-sucedida dos sistemas, sendo concretizada em rotinas realizadas pelas equipes de desenvolvimento dos sistemas. Assim, uma demanda por implementação de uma nova funcionalidade do sistema (um aprimoramento), por exemplo, é formada pelas atividades de direcionamento da demanda para um profissional, implementação, testes locais, integração com o restante do código do sistema, 
homologação que consiste na validação do código integrado, testes gerais realizados por um setor de controle de qualidade e disponibilização do aprimoramento para os usuários.

Cada equipe possui uma coordenação específica e está subordinada à diretoria de sistemas. Segundo o entrevistado E2 que enfatiza a importância dos processos de trabalho realizados e ações coordenadas:

O setor de TI hoje existe porque ele possui essa capacidade técnica, que é organizacional. As equipes, elas interagem entre si, são equipes que tem conhecimento de sua área, mas que se complementam... existe processo e metodologia definidos para sustentar essas coisas. Existe o pensamento de que cada um faz o seu, mas que junto a gente consegue fazer as entregas tanto internas quanto externas (E2).

Neste contexto, a capacidade técnica de Tecnologia da Informação é reconhecida como uma habilidade organizacional em executar duas atividades principais: desenvolver e disponibilizar novas funcionalidades para os sistemas integrados e corrigir erros destes sistemas. Cada uma destas atividades é tratada de maneira específica, conforme dito por um dos coordenadores de equipe.

\footnotetext{
A demanda chega pra gente através dos setores de suporte, requisitos. A gente analisa que pessoa pode fazer em tempo hábil ou que tenha maior familiaridade com as regras de negócios. Quanto às demandas lá de suporte, a maioria são erros e pequenos aprimoramentos. [...] Já projetos maiores e de fluxos maiores então a equipe de requisitos fica responsável, cataloga tudo, faz reuniões com clientes, gera requisitos e com isso em mãos conversa-se comigo [...] e a gente dá o encaminhamento e também os prazos, deadlines, porque transforma já num projeto mesmo (E11).
}

Quanto ao desempenho relacionado às atividades de desenvolvimento dos sistemas inerente à capacidade técnica, realizá-las no tempo definido e a entrega de um produto de qualidade são dois indicadores relevantes para capacidade técnica, conforme exposto a seguir, por membros das equipes de suporte e requisitos respectivamente (E9 e E1).

[...] a gente procura atender em um período, não passar muito tempo. Para demanda de erros o prazo de atendimento é de quarenta e oito horas: tem um período, assim, quando a demanda é urgente (erros). Geralmente o prazo é atendido (E9).

Eu identifico que nossa equipe de suporte e de requisitos, como ela é o "para-choque" [...] então a partir do momento que o meu back-end ali, ou seja, a equipe de desenvolvimento, ele produz algo que não está dentro do padrão que o usuário quer, isso vai refletir na gente também... o usuário é nosso cliente e nós somos clientes de desenvolvimento. (E1).

Considerando o tempo de atendimento como um importante indicador de evolução da capacidade técnica, a figura 1 a seguir exibe o prazo médio em dias da realização de tarefas pelas equipes de desenvolvimento, entre o segundo semestre de 2010 e o segundo semestre de 2013. Este gráfico exibe uma tendência de queda neste prazo, demonstrando melhorias no desempenho desta capacidade.

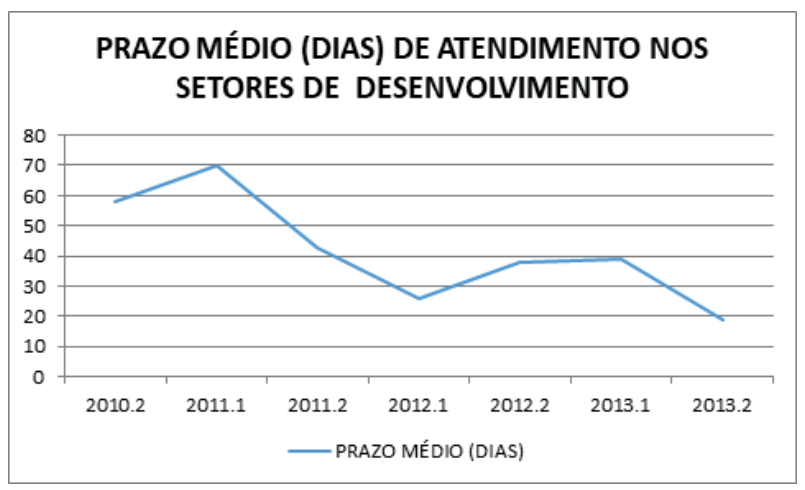

Figura 1 Tempo médio de atendimento de atividades de desenvolvimento entre 2010 e 2013.

Fonte: Dados da Pesquisa (2014)

Diante disso, define-se capacidade técnica como uma habilidade organizacional em desenvolver e disponibilizar novas funcionalidades e correções de erros dos sistemas integrados para os clientes internos e externos a partir das demandas das unidades de suporte e requisitos em tempo e qualidade esperados, através do direcionamento para um membro da equipe, implementação, testes, integração, homologação e disponibilização do aprimoramento ou correção.

Esta definição estende àquelas apresentadas pela literatura que negligenciam quatro características relevantes para uma capacidade: habilidade organizacional, coordenação, rotinas e desempenho. Quanto à primeira característica, enriquece a defi- 
nição ao sugerir esta capacidade como disseminada na organização (HELFAT; PETERAF, 2003) e não apenas baseada em um know how (PICCOLI; IVES, 2005; BARNEY; CLARK, 2007; HULLAND ET AL., 2007), o que pressupõe conhecimento localizado unicamente no profissional, bem como indica que as tarefas envolvidas devem pressupor uma coordenação entre unidades distintas (setor de desenvolvimento do software e as unidades que demandam).

Outro aspecto negligenciado na literatura é a ausência das rotinas que compõem a capacidade técnica. Neste sentido, tal rotina é apresentada na definição (COLLIS, 1994; DOSI ET AL., 2000; WINTER, 2000; HELFAT; PETERAF, 2003), necessário para maior aproximação e concretização da capacidade com os seus envolvidos (SALVATO; RERUP, 2011). Por fim, a definição acima ainda sugere a adoção de indicadores tempo e qualidade para gestão mais efetiva da capacidade e em busca de um desempenho confiável (SCHREYÖGG; KLIESCH-EBERL, 2007), aspecto também não apontado na literatura.

\subsection{Capacidade de Relacionamento Interno}

Ao se relacionar com usuários dos sistemas integrados da UFRN, o setor de Tecnologia da Informação construiu uma capacidade de interagir com estes atores (professores e servidores técnicos) de uma maneira específica (ETHIRAJ ET AL., 2005).

Em geral, a rotina que caracteriza a concretização desta capacidade consiste no recebimento, acompanhamento e retorno de uma demanda por esclarecimento de dúvidas, correção de erros ou requisições de serviços pelos usuários. Eventualmente, há a necessidade de reuniões com os usuários para melhor compreensão de suas necessidades e estruturação deste conhecimento para ser melhor executado pelas equipes de desenvolvimento.

Esta capacidade é analisada principalmente pelos dois setores que diretamente interagem com estes atores, conforme citam os dois entrevistados a seguir, pertencentes a estes setores da unidade de Tecnologia da Informação (E7 e E1):
[...] A TI é confiável ao ponto de ser demandada, e (é uma capacidade) coordenada pela equipe de requisitos junto com os coordenadores pra dar o aval técnico. Estão envolvidos nisto os coordenadores e analista de requisitos (E7).

[...] a gente mantém reuniões, a equipe de requisitos está sempre em contato com os gestores pra tentar coletar, colher ou entender, responder alguma dúvida ou necessidade que deva ser incorporada ao sistema, então tem essa capacidade, habilidade, acredito que organizacional de absorver essa necessidade... e são coordenadas (E1).

Um dos usuários internos da IES, que demanda constantemente correções de erros e novos aprimoramentos no sistema atesta esta capacidade de relacionamento interno, bem como o reconhecimento da qualidade já "conquistada", conforme descrito pelo entrevistado E17.

[...] acho que a equipe hoje tem essa maturidade também, que também foi sendo construída. E a gente chegou a essa qualidade [...] porque até acho que o compromisso hoje é de no mínimo manter. Porque todo mundo que tá lá sabe... a gente tem planos de melhorias, tem muita coisa pra implementar, mas tem um mínimo, um padrão SIG que foi estabelecido. (E17).

O tempo de atendimento é também um importante indicador relacionado ao desempenho das atividades de relacionamento interno, já que influencia diretamente nas atividades diárias dos usuários, conforme atesta um membro do setor de suporte (E1):

\begin{abstract}
Se é um erro de execução a gente tá impedindo um usuário que ele execute o trabalho dele. E aí, o que acontece? o usuário, ele tem uma data limite, por exemplo, se for questão de um módulo financeiro, orçamentário, que tem um limite pra empenhar alguma coisa, um orçamento, então ele diz: "eu tenho até dia 20 pra empenhar" se dia 19 der um erro, tem que ser consertado (E1)!
\end{abstract}

Tendo em vista o prazo de atendimento como importante indicador, a figura 2 exibe prazo médio de atendimento considerando as correções de erros, mais rápidas de serem realizadas, como as implementações de novas funcionalidades, que demandam um tempo maior. É possível verificar uma tendência de diminuição nesta variável. 


\section{PRAZO MÉDIO (DIAS) DE ATENDIMENTO NO SETOR DE SUPORTE}

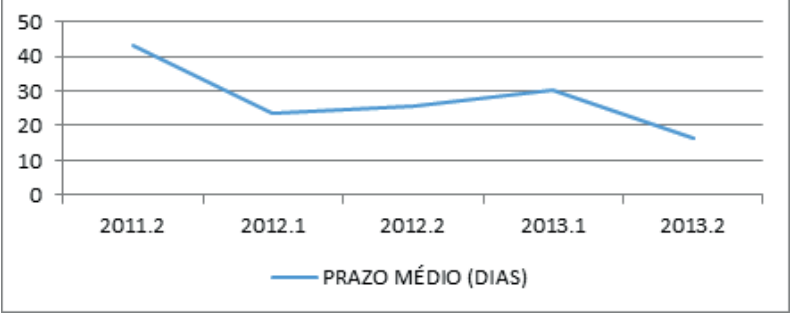

Figura 2 Tempo médio de atendimento interno aos usuários da IES entre 2011 e 2013.

Fonte: Dados da Pesquisa (2014)

Dessa maneira, capacidade de relacionamento interno é definida como habilidade organizacional em apoiar o uso efetivo dos sistemas integrados pelos clientes institucionais em suas atividades, ao atender demandas dos gestores por alterações relevantes, como também da comunidade da IES em geral por dúvidas, correções e melhorias, através de reuniões com usuário, recepção, acompanhamento e retorno da demanda em tempo e qualidade esperados.

Dessa forma, as características de desempenho adequado (tempo e qualidade) (SCHREYÖGG; KLIESCH-EBERL, 2007) e descrição da rotina (COLLIS, 1994; DOSI ET AL., 2000; WINTER, 2000; HELFAT; PETERAF, 2003) são duas características poucos salientes na literatura, mas explicitado pela definição acima.

\subsection{Capacidade de Cooperação externa}

Como capacidade mais recente a ser construída, em decorrência do relacionamento entre a IES e as instituições cooperadas, a cooperação externa apresenta os maiores desafios na gestão. Esta é caracterizada por rotinas similares àquelas realizadas pela capacidade de relacionamento interno, com algumas variações como realizações de treinamentos a distância, através de videoconferências, e execução de algumas intervenções técnicas no ambiente operacional da instituição cooperada.

O entrevistado E10, coordenador de uma das equipes de desenvolvimento, explicita a seguir um destes desafios, relacionados à decisão da UFRN em implementar melhorias nos sistemas que sejam benéficas para a rede de cooperação.

\begin{abstract}
A entrega é uma resposta às demandas deles. E nem sempre coisas que são demandadas são feitas, até porque a gente só desenvolve coisas que sejam benéficas pra toda rede. Verdade que pode ter alguma demanda específica. Mas assim, eu acredito que é [...] uma gerência de relação, o fato da gente tá negando não impede que essa relação seja feita. Nosso papel é fazer um modelo de referência e se isso não é uma referência possível pras outras, estaria fora do escopo (E10).
\end{abstract}

Neste modelo de relacionamento, as instituições têm a responsabilidade em implantar os sistemas, mas a IES precisa dar suporte, tanto disponibilizando os sistemas em versões atualizadas (nos chamados ambientes de referências disponíveis para cada instituição), para que as instituições tenham condições de acessá-lo e incorporá-lo em sua infraestrutura, caso desejem, quanto esclarecendo dúvidas de natureza técnicas ou negociais.

Além disso, de maneira similar às atividades de relacionamento interno, os atendimentos às instituições cooperadas são por correções de erros e por aprimoramentos. Ao justificar a importância de respostas no tempo adequado, o entrevistado E15, gestor de Tecnologia da Informação de uma instituição cooperada presentes desde os primeiros termos de cooperação, atesta:

[...] quando a gente gera um chamado pra lá é porque a gente já tá recebendo um chamado interno [...] se nós estamos solicitando algo foi porque alguém interno nosso solicitou. [...] Ou seja, o nosso usuário final vai esperar num tempo muito maior [...] além do nosso tempo de solicitação, tem o tempo da [IES]. E se eu não posso dar pro meu usuário final uma expectativa de tempo, aí eu gero insatisfação. É uma pessoa a menos, que não quer usar o sistema, se é alguém que tá numa posição de gestão pode ser que... seja alguém que tenha o poder de dizer: "ó, não tá funcionando esse negócio, vamo parar!” Então isso tem consequências (E15).

A figura 3 exibe o tempo médio de atendimento às demandas das instituições, por erros ou aprimoramentos nos sistemas integrados, demonstrando uma tendência de queda neste prazo médio, a partir da perspectiva do setor de requisitos entre os anos de 2011 e 2013. 


\section{PRAZO MÉDIO (DIAS)}

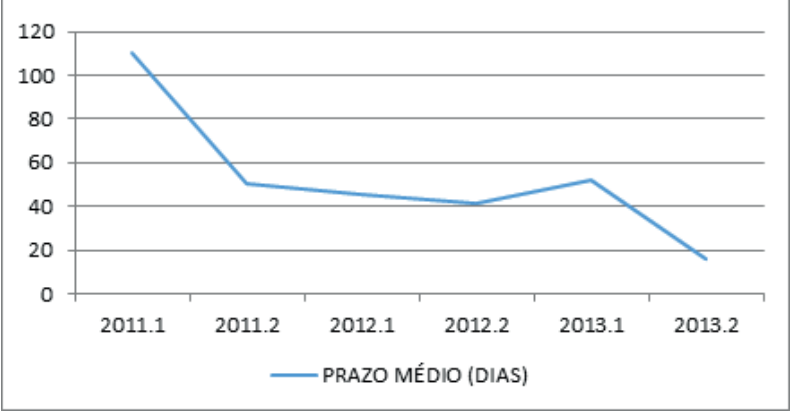

Figura 3 Tempo médio de atendimento às instituições cooperadas entre 2011 e 2013. Fonte: Dados da Pesquisa (2014).

A capacidade de cooperação externa diz respeito à habilidade organizacional de transferir conhecimento para as instituições cooperadas através do atendimento às suas demandas, seja por acesso, correções e melhorias nos sistemas de referência, benéficas para as redes de cooperação; seja por esclarecimento de dúvidas e treinamentos de natureza negocial e técnica, em tempo e qualidade esperados.

Para esta capacidade, a característica relacionada à necessidade de desempenho adequado (SCHREYÖGG; KLIESCH-EBERL, 2007) foi identificada na literatura como ausente e incorporada na definição. Assim como nas demais capacidades, esta característica possibilita uma gestão mais efetiva do relacionamento externo, ao possibilitar monitoramento e controle que identifique casos em que o desempenho esteja inferior a um nível de atendimento adequado e ações sejam tomadas para melhoria no desempenho.

\section{CONSIDERAÇÕES FINAIS}

O presente trabalho identificou as capacidades de TI relevantes para concepção, implantação e disseminação dos sistemas integrados tanto na IES quanto em instituições do governo federal cooperadas. Como resultado, foi possível identificar as capacidades técnica, de relacionamento interno e de cooperação externa como relevantes neste contexto.

De modo geral, pode-se inferir que estas capacidades identificadas são concretizadas através de atividades diárias responsáveis pela operacionalização das atividades envolvendo usuários internos (UFRN) ou externos (instituições parceiras). Dessa forma, apenas capacidades caracterizadas como operacionais ou ordinárias foram identificadas (Winter, 2003).

Ao relacionar as características conceituais de uma capacidade organizacional às definições dos tipos de capacidades de Tecnologia da Informação da literatura e instanciá-los ao caso em questão, foi possível elaborar definições mais robustas das capacidades de Tecnologia da Informação no caso, principalmente capacidade técnica, possibilitando um avanço e operacionalização destas definições, e consequentemente maior possibilidade de criar, construir e gerenciar tais capacidades de acordo com as intenções estratégicas das instituições.

Assim, não houve unanimidade na identificação de capacidades dinâmicas no contexto da trajetória dos sistemas integrados da IES, como capacidade de responder ao ambiente ou capacidade de planejamento e mudanças, relevantes para integrar, reconfigurar, renovar ou recriar novos recursos e capacidades em resposta a mudanças ambientais. Capacidades desta natureza são importantes para a sobrevivência organizacional em curto prazo, mas precisam ser gerenciadas e alteradas apropriadamente através de mecanismos fornecidos por outro tipo de capacidade: a dinâmica, sejam funcionais ou de aprendizado (HINE ET AL., 2014), pois permite minimizar o risco de ter as capacidades operacionais tornadas irrelevantes (TEECE, 2007; HELFAT ET AL., 2009; YEPES, 2006).

Esta não identificação das cinco capacidades pode ter ocorrido principalmente pela ausência na percepção dos respondentes sobre uma rotina estabelecida que indique a existência delas. Alguns participantes até expressaram reconhecer ações pontuais relacionadas à capacidade (WINTER, 2003), mas não de forma consistente. Isso indica a necessidade em se desenvolver um desempenho mais confiável para a instituição e a importância das rotinas enquanto construtos relevantes para capacidades (COLLIS, 1994; DOSI ET AL., 2000; WINTER, 2000; HELFAT; PETERAF, 2003).

Enquanto limitações da pesquisa, ressalta-se o caráter dedutivo em que foram identificadas as capacidades de Tecnologia da Informação pelos respondentes, já que tais capacidades foram limitadas 
por aquelas propostas pela literatura, limitando a possibilidade de identificação de outras. Além disso, dada a impossibilidade de acesso durante a pesquisa, não foi possível entrevistar gestores a respeito da identificação das capacidades de Tecnologia da Informação, limitando tal percepção a um contexto interno dos membros da Tecnologia da Informação da instituição.

Enquanto perspectiva futura, espera-se que esta pesquisa possa ser adotada como referência para identificação de capacidades de Tecnologia da Informação em outros contextos organizacionais, enquanto uma etapa relevante para que capacidades não reconhecidas, mas estratégicas, sejam apropriadamente desenvolvidas ao longo do tempo.

\section{REFERÊNCIAS}

AGUILAR ZAMBRANO, José Javier; YEPES, Edgar Rene. Gestión de capacidades dinámicas e innovación: una aproximación conceptual. Revista de Ciências da Administração, v. 8, n. 16, 2006.

AMIT, R.; SCHOEMAKER, P. J. Strategic assets and organizational rent. Strategic management journal, v. 14, n. 1, p. 33-46, 1993.

ANDREWS, K. The Concept of Corporate Strategy. Homewood, IL. 1971

ARAL, S.; WEILL, P. IT assets, organizational capabilities, and firm performance: How resource allocations and organizational differences explain performance variation. Organization Science, v. 18, n. 5, p. 763-780, 2007.

BARNEY, J. B.; CLARK, D. N. Resource-based theory: Creating and sustaining competitive advantage. Oxford: Oxford University Press Oxford, 2007.

BHARADWAJ, A. A resource-based perspective on information technology capability and firm performance: an empirical investigation. MIS Quarterly, p. 169-196, 2000.
BHARADWAJ, A.; SAMBAMURTHY, V.; ZMUD, R. W. IT capabilities: theoretical perspectives and empirical operationalization. Proceedings of the 20th international conference on Information Systems, Association for Information Systems. p.378-385, 1999.

BHATT, G. et al. Building and leveraging information in dynamic environments: The role of IT infrastructure flexibility as enabler of organizational responsiveness and competitive advantage. Information \& Management, v. 47, n. 7, p. 341-349, 2010 .

BHATT, G. D.; GROVER, V. Types of information technology capabilities and their role in competitive advantage: an empirical study. Journal of management information systems, v. 22, n. 2, p. 253-277, 2005.

BYRD, T. A.; TURNER, D. E. An exploratory examination of the relationship between flexible IT infrastructure and competitive advantage. Information \& Management, v. 39, n. 1, p. 41-52, 2001.

CARR, N. G. TI já não importa. Harvard business review, v. 81, n. 5, p. 30-37, 2003.

COLLIS, D. J. Research note: how valuable are organizational capabilities? Strategic management journal, v. 15, n. 1, p. 143-152, 1994.

DAY, G. S. The capabilities of market-driven organizations. the Journal of Marketing, p. 37-52, 1994.

DOSI, G.; NELSON, R.; WINTER, S. The nature and dynamics of organizational capabilities. Oxford University Press, 2000.

DUNCAN, N. B. Capturing Flexibility of Information Technology Infrastructure: A Study of Resource Characteristics and their Measure. Journal of management information systems, v. 12, n. 2, p. 37-57, 1995. 
ETHIRAJ, S. K. et al. Where do capabilities come from and how do they matter? A study in the software services industry. Strategic management journal, v. 26, n. 1, p. 25-45, 2005.

FEENY, D. F.; WILLCOCKS, L. P. Core IS capabilities for exploiting information technology. Sloan management review, v. 39, n. 3, p. 9-21, 1998.

FELDMAN, M. S.; PENTLAND, B. T. Reconceptualizing organizational routines as a source of flexibility and change. Administrative science quarterly, v. 48, n. 1, p. 94-118, 2003.

FINK, L.; NEUMANN, S. Exploring the perceived business value of the flexibility enabled by information technology infrastructure. Information \& Management, v. 46, n. 2, p. 90-99, 2009.

GIBBS, G. Análise de Dados Qualitativos. Porto Alegre. 2009

GIL, A. C. Como elaborar projetos de pesquisa. São Paulo, v. 5, 2002.

GODOY, A. S. Estudo de caso qualitativo. In: SARAIVA (Ed.). Pesquisa Qualitativa em Estudos Organizacionais: paradigmas, estratégias e métodos. São Paulo: Saraiva, 2006.

GRANT, R. M. Contemporary strategy analysis and cases: text and cases. Wiley, 2010.

HARRISON, J. S. et al. Resource complementarity in business combinations: Extending the logic to organizational alliances. Journal of management, $\mathrm{v}$. 27, n. 6, p. 679-690, 2001.

HELFAT, C. E. et al. Dynamic capabilities: Understanding strategic change in organizations. Wiley. com, 2009.

HELFAT, C. E.; PETERAF, M. A. The dynamic resource-based view: capability lifecycles. Strategic management journal, v. 24, n. 10, p. 997-1010, 2003.
HELFAT, C. E.; WINTER, S. G. Untangling Dynamic and Operational Capabilities: Strategy for the $(\mathrm{N})$ ever-Changing World. Strategic management journal, v. 32, n. 11, p. 1243-1250, 2011.

HINE, D.; PARKER, R.; PREGELJML.; VERREYNNE, N. Deconstructing and reconstructing the capability hierarchy. Industrial and Corporate Change, v. 23, n. 5, p. 1299-1325, 2014.

HULLAND, J.; WADE, M. R.; ANTIA, K. D. The impact of capabilities and prior investments on online channel commitment and performance. Journal of management information systems, v. 23, n. 4, p. 109-142, 2007.

KEEN, P. G. W. Information technology and the management difference: a fusion map. IBM Systems Journal, v. 32, n. 1, p. 17-39, 1993.

LEINWAND, P.; MAINARDI, C. The coherence premium. Harvard Business Review, v. 88, n. 6, p. 86-92, 2010.

LEVINTHAL, D. Organizational capabilities in complex worlds. The nature and dynamics of organizational capabilities, p. 363-379, 2000.

LIANG, T.-P.; YOU, J.-J.; LIU, C.-C. A resource-based perspective on information technology and firm performance: a meta analysis. Industrial Management \& Data Systems, v. 110, n. 8, p. 11381158, 2010.

MAKADOK, R. Toward a synthesis of the resource-based and dynamic-capability views of rent creation. Strategic management journal, v. 22, n. 5, p. 387-401, 2001.

MATA, F. J.; FUERST, W. L.; BARNEY, J. B. Information technology and sustained competitive advantage: a resource-based analysis. MIS Quarterly, p. 487-505, 1995. 
MELVILLE, N.; KRAEMER, K.; GURBAXANI, V. Review: Information technology and organizational performance: An integrative model of IT business value. MIS Quarterly, v. 28, n. 2, p. 283-322, 2004.

MILES, M. B.; HUBERMAN, A. M. Qualitative data analysis: An expanded sourcebook. Sage, 1994. ISBN 0803955405.

MONTEALEGRE, R. A Process Model of Capability Development: Lessons from the Electronic Commerce Strategy at Bolsa de Valores de Guayaquil. Organization Science, v. 13, n. 5, p. 514-531, 2002.

NAVARRO, Jose Ruiz; GALLARDO, Francisca Orihuela. A model of strategic change: Universities and dynamic capabilities. Higher education policy, v. 16, n. 2, p. 199-212, 2003.

NELSON, R. R.; WINTER, S. An evolutionary theory of economic change. Harvard University Press, 1982.

NEVO, S.; WADE, M. Firm-level benefits of ITenabled resources: A conceptual extension and an empirical assessment. The Journal of Strategic Information Systems, v. 20, n. 4, p. 403-418, 2011.

NEVO, S.; WADE, M. R. The formation and value of IT-enabled resources: antecedents and consequences of synergistic relationships. MIS Quarterly, v. 34, n. 1, p. 163-183, 2010.

PENROSE, E. T. A teoria do crescimento da firma. Ed. Unicamp, 2006.

PEREIRA, M.; FORTE, S. Visão baseada em recursos nas instituições de ensino superior de Fortaleza: uma análise ex-ante e ex-post à LDB/96. In: ENCONTRO ANUAL DA AS- SOCIAÇÃO NACIONAL DE PÓSGRADU- AÇÃO E PESQUISA EM ADMINISTRAÇÃO, 28., 2004, Curitiba. Anais... Curitiba: EnANPAD, 2004.
PICCOLI, G.; IVES, B. Review: IT-dependent strategic initiatives and sustained competitive advantage: a review and synthesis of the literature. MIS Quarterly, v. 29, n. 4, p. 747-776, 2005.

PREGELJ, L. Capability development along the capability lifecycle: Evidence from the pharmaceutical industry. 2013. 316 (Tese de Doutorado). UQ Business School, The University of Queensland Austrália.

RAHMANDAD, Hazhir; REPENNING, Nelson. Capability erosion dynamics. Strategic Management Journal, v. 37, n. 4, p. 649-672, 2016.

RAPP, A.; TRAINOR, K. J.; AGNIHOTRI, R. Performance implications of customer-linking capabilities: Examining the complementary role of customer orientation and CRM technology. Journal of Business research, v. 63, n. 11, p. 1229-1236, 2010.

RAVICHANDRAN, T.; LERTWONGSATIEN, C. Effect of information systems resources and capabilities on firm performance: a resource-based perspective. Journal of management information systems, v. 21, n. 4, p. 237-276, 2005.

RICHARDSON, G. B. The organisation of industry. The economic journal, v. 82, n. 327, p. 883-896, 1972.

ROSS, J. W.; BEATH, C. M.; GOODHUE, D. L. Develop long-term competitiveness through IT assets. Sloan management review, v. 38, n. 1, p. 3142, 1996.

SABHERWAL, Rajiv; KIRS, Peeter. The alignment between organizational critical success factors and information technology capability in academic institutions. Decision Sciences, v. 25, n. 2, p. 301330, 1994.

SALVATO, C.; RERUP, C. Beyond collective entities: Multilevel research on organizational routines and capabilities. Journal of management, v. 37, n. 2, p. 468-490, 2011. 
SCHREYÖGG, G.; KLIESCH-EBERL, M. How dynamic can organizational capabilities be? Towards a dual-process model of capability dynamization. Strategic management journal, v. 28, n. 9, p. 913-933, 2007. ISSN 1097-0266.

SIRMON, D. G.; HITT, M. A.; IRELAND, R. D. Managing firm resources in dynamic environments to create value: Looking inside the black box. Academy of management Review, v. 32, n. 1, p. 273-292, 2007.

STIEGLITZ, N.; HEINE, K. Innovations and the role of complementarities in a strategic theory of the firm. Strategic management journal, v. 28, n. 1, p. 1-15, 2007.

STOEL, D. M.; MUHANNA, W. A. IT capabilities and firm performance: A contingency analysis of the role of industry and IT capability type. Information \& Management, v. 46, n. 3, p. 181-189, 2009.

TEECE, D. J. Explicating dynamic capabilities: the nature and microfoundations of (sustainable) enterprise performance. Strategic management journal, v. 28, n. 13, p. 1319-1350, 2007.

TEECE, D. J.; PISANO, G.; SHUEN, A. Dynamic capabilities and strategic management. Strategic management journal, v. 18, n. 7, p. 509-533, 1997.

ULRICH, D.; SMALLWOOD, N. Capitalizing on capabilities. Harvard business review, p. 119-128, 2004.

WADE, M.; HULLAND, J. Review: The resource-based view and information systems research: Review, extension, and suggestions for future research. MIS Quarterly, v. 28, n. 1, p. 107-142, 2004.

WINTER, S. G. The satisficing principle in capability learning. Strategic management journal, v. 21, n. 10-11, p. 981-996, 2000.

Understanding dynamic capabilities. Strategic management journal, v. 24, n. 10, p. 991 995, 2003.
YIN, R. K. Case study research: Design and methods. Ed. Sage, 2003.

ZOLLO, M.; WINTER, S. G. Deliberate Learning and the Evolution of Dynamic Capabilities. Organization Science, v. 13, n. 3, p. 339-351, 2002. Disponível em: < http://pubsonline.informs.org/doi/ abs/10.1287/orsc.13.3.339.2780 > . 